\title{
Research on the Principles and Strategies of Cultivating the Sports Core Quality of Students in Higher Vocational Colleges
}

\author{
Xiaoqin He, Chunquan Zhu* \\ Sports Department, Sichuan Vocational and Technical College, Suining, China \\ Email address: \\ 155389260@qq.com (Xiaoqin He), 1010119554@qq.com (Chunquan Zhu) \\ ${ }^{*}$ Corresponding author
}

To cite this article:

Xiaoqin He, Chunquan Zhu. Research on the Principles and Strategies of Cultivating the Sports Core Quality of Students in Higher Vocational Colleges. International Journal of Sports Science and Physical Education. Vol. 5, No. 3, 2020, pp. 35-40. doi: 10.11648/j.ijsspe.20200503.12

Received: September 27, 2020; Accepted: October 9, 2020; Published: October 14, 2020

\begin{abstract}
Under the guidance of China's policy of attaching great importance to the cultivation of socialist builders and successors, the task of higher vocational colleges is to train students with certain higher education knowledge and professional skills under the general requirements of "Literal cultivation". Under this goal, how the sports curriculum reform of higher vocational colleges occupy an important position in the talent training system and support the sports core quality in the training process of students. This depends on the cultivation and the construction of the sports core qualities. This paper is based on the definition of the core literacy of the system. From the perspective of discovering and solving problems, it faces the problems encountered in the research process of sports core literacy, analyzes the causes, and studies the training principles and training strategies from the problems. The core literacy training principles and strategies are used to continuously enrich the research standards and framework of the core literacy of the sports discipline, make the system and structure of the sports core literacy more in line with the requirements of curriculum reform, and broaden the training ideas of the core literacy of the students in higher vocational colleges to meet the country and society's demand for talents.
\end{abstract}

Keywords: Higher Vocational Colleges, Sports Core Literacy, Problems, Principles and Strategies

\section{Preface}

\subsection{Introduction}

"If the youth prospers, the country will prosper; if the youth is strong, the country will be strong." According to the requirements of moral education, higher vocational colleges should focus on cultivating students' sports core quality and enhancing their physical quality. It is an important mission of PE teachers in Higher Vocational Colleges in the new era to analyze the problems of sports core literacy in Higher Vocational Colleges and accelerate the research and cultivation of sports core literacy in higher vocational colleges. The cultivation of sports core literacy plays an important role in helping students form a good habit of actively participating in sports activities, cultivating the excellent quality of hard work and perseverance, and cultivating talents needed by social development. With the fierce market competition, the requirements for talents are also improving. Students with core sports demand can be more competitive in the society, laying the foundation for the overall development of individuals.

\subsection{Research Background}

January 2019, the State Council issued the implementation plan of national vocational education reform, which requires: "vocational education should implement the fundamental task of moral education, improve the education mechanism of combining morality and technology, work and study, improve the evaluation mechanism, and standardize the whole process of talent training. [1] November 2019, the decision of the Central Committee of the Communist Party of China on upholding and improving the socialist system with Chinese characteristics and promoting the modernization of the 
national governance system and governance capacity, which was deliberated and adopted by the Fourth Plenary Session of the 19th CPC Central Committee, pointed out that "we should improve the system and mechanism of 'Cultivating morality and cultivating talents', deepen the comprehensive reform in the field of education, and cultivate' morality, intelligence, physique and beauty ' Labor is a socialist builder and successor with all-round development. [2]". Under the policy guidance of the Party Central Committee and the State Council, under the premise of "cultivating morality and cultivating people" and comprehensively promoting the "three education reform", the physical education curriculum in higher vocational colleges will follow and grasp the core elements of physical education curriculum and teaching reform, focus on cultivating students' Sports core literacy, improving their sports morality and cultivation, sports interest and ability, healthy behavior and habits, and sports quality [3], form the consciousness, habit and ability of lifelong physical exercise, promote the comprehensive and coordinated development of students, work healthily, and contribute to social progress.

\subsection{Literature Review}

At present, the elaboration of sports core literacy in sports field is not mature. From the perspective of research status, the core literacy in sports field is mainly concentrated in basic education, and the research results on University Sports Core literacy are less. Sports core literacy belongs to the category of core literacy. Compared with the core literacy of sports discipline, the scope of sports core literacy is more extensive. Sun Hong (2019) believes that the framework of College Students' Sports Core literacy is divided into three dimensions: Sports tools, sports practice and sports values [4]; Zhan Limin (2019) believes that the core quality of university sports includes six qualities: knowledge of health promotion, behavior of health promotion, ability of independent sports, habit of conscious exercise, attitude of self-education and self-improvement character [5]; Hou Jie (2020) believes that firstly, the elements of college sports core literacy are constructed theoretically, and then the elements are defined by Delphi method. Finally, it is determined that the core sports literacy of college students is composed of 6 first level elements and 23 second level elements. The first level elements include sports ability, sports knowledge, health behavior, sports morality, sports values and sports information literacy [6]. According to the comprehensive academic research, students' Sports Core literacy is a comprehensive sports literacy, including sports morality and cultivation, sports interest and ability, healthy behavior and habits, sports quality and will, covering ability, knowledge, values, attitude, emotion and other contents [7]. It has subjectivity and difference, that is, whether the connotation of the cultivation of sports core literacy of the same individual in different periods is different In the same way, different individuals have different ways to cultivate sports core literacy.

\section{The Meaning of Sports Core Literacy}

The core quality is the embodiment of the party's educational policy and the intermediate link connecting the macro educational concept, training objectives and specific education and teaching practice. Through the bridge of core literacy, the party's education policy can be transformed into specific requirements that can be used in education and teaching practice and can be easily understood by educators, so as to clarify the essential characters and key abilities that students should possess. The core quality of sports is a comprehensive sports quality. Its purpose is to establish the physical basis and emotional cognition of the essential character and key ability to meet the needs of life-long development and social development, including sports morality and cultivation, sports interest and ability, healthy behavior and habits, sports quality and will, etc. For teenagers, the goal of sports core literacy training is to master basic sports knowledge and basic sports skills; for college students who are about to enter the society to participate in work, the goal of sports core literacy should be to develop sports health literacy, sports spirit, sports cultural heritage, etc. [8]. Sports core literacy is the development and upgrading of sports literacy. It pays more attention to the key literacy that students need to adapt to life-long development in future social life. It advocates that students need to master sports skills, at the same time, they should further enhance their physical health and cultivate sports social emotions, form a certain sports interest and skills, learn basic sports health knowledge and behavior, and establish good sports To develop the habit of lifelong exercise.

\section{Problems in the Cultivation of Students' Sports Core Quality in Higher Vocational Colleges}

\subsection{Ideologically Ignorance}

The cultivation of students' Sports Core literacy plays an important role. However, in the work of higher vocational colleges, there is a problem of not paying attention to the core quality of sports, which is reflected in the following two aspects: first, in terms of students, influenced by the traditional examination oriented education, primary and secondary school students mainly concentrate on the study of cultural courses, and invest less energy in sports in university, not paying any attention to the physical education curriculum and physical exercise. Many college students indulge in the network, games, love and so on in their spare time, leading to the deterioration of students' physical quality. Second, in the school, the existing evaluation index of colleges and universities is not reasonable in the evaluation of students' academic performance. For example, the scholarship usually goes to the top students in the class but the weight of physical quality assessment seldom be considered, which leads to that the physical education teachers and students do not pay attention to the development of physical fitness and sports 
ability. In terms of knowledge popularization, sports knowledge, injury prevention, first-aid lectures and other activities held by higher vocational colleges are also relatively few, and relevant experts and scholars are rarely invited to carry out necessary science popularization and explanation, so that students' sports professional knowledge and understanding level is not high.

\subsection{Imperfect Curriculum System}

There are relatively few physical education courses in higher vocational colleges. Generally speaking, physical education is held once or twice in a week. At the same time, the teaching content is relatively simple. There are many traditional physical education courses, but only a few school-based courses and elective activities. The final assessment is mainly to investigate the relevant skills of students, rather than students' implicit ability. Meanwhile, physical education is usually limited in the campus, but not widely extended to the outside. The construction of perfect classroom system can promote the cultivation of students' Sports Core literacy. Therefore, higher vocational colleges need to improve the existing curriculum system to lay a foundation for the cultivation of sports core literacy.

\subsection{The Construction of Teaching Staff Needs to Be Strengthened}

The quality of physical education teachers will have a certain impact on the cultivation of core literacy. At present, the construction of sports teachers in higher vocational colleges is slow. First of all, higher vocational colleges have not carried out necessary special training on Sports Core literacy for physical education teachers, and many physical education teachers have not received systematic training. Some higher vocational colleges put forward special funds for PE teacher training, but they did not carry out the corresponding ability evaluation before the training, so that the training activities are lack of pertinence, resulting in a waste of funds. What's more, some PE teachers don't pay attention to their professional development in their spare time, so that their ability is improved slowly, which has brought some obstacles to the development of sports core training. Most physical education teachers can not understand the core quality of physical education from the cognitive level, students' growth, constituent elements, training objectives, practical approaches and evaluation indicators.

\subsection{Campus Sports Atmosphere Needs to Be Optimized}

School is the main place for students to spend most time at, and the campus atmosphere of higher vocational colleges will also have an impact on the cultivation of students' Sports Core literacy. At present, the school's material investment in sports hardware resources is not enough, and the amateur activities is relatively few. Some colleges and universities sports activities and sports events are limited to traditional basketball, football, badminton, etc.. Students' sports life is not rich enough, so it is difficult to attract students to participate, and the construction of some sports associations is relatively slow. What's more serious is that due to the heavy task of teaching cultural courses, some schools have a busy curriculum, and even arrange classes on weekends and evening. Some teachers use the after-school exercise time to ask students to study or do experiment, so that the students' spare time for exercise is shortened or occupied.

\section{Principles and Strategies of Cultivating students' Sports Core Quality in Higher Vocational Colleges}

\subsection{Cultivation Principles of Students' Sports Core Literacy}

\subsubsection{Principle of Difference}

Different students have different characteristics. There are many differences between students, including gender differences, physical fitness differences and sports interest differences, etc.. The existence of these differences makes students' Sports Core literacy uneven [9]. In order to make the training activities more targeted and focused, it is necessary to base on the actual situation of students when cultivating students' Sports Core literacy. Following the principle of individual differences, colleges and universities need to do a good job of finding out the truth, investigate the situation of students' Sports Core literacy, and formulate training strategies combined with the specific situation, so as to improve the training effect of sports core literacy. For example, a network database is established for the basic information of students, such as age, height, weight and sports level, so as to update and monitor the physical condition of students in real time. Big data analysis is used to classify students reasonably, set up physical education courses according to different types, and adopt different training methods.

\subsubsection{Systematic Principle}

The cultivation of sports core literacy is a systematic project, which not only needs the participation of schools and teachers, but also needs the participation of parents and students. In terms of schools, they needs to do a good job in the construction of physical education curriculum, and organically combine the discipline core literacy with the training objectives. In terms of parents, they need to carry out necessary education for students to make them realize the importance of sports core literacy training for their own development. In terms of students, they can reflect their own needs to teachers, who can summarize the needs of students, combined with the specific needs of students and teaching content to carry out corresponding sports activities and reduce the workload of teachers.

\subsubsection{Principle of Effectiveness}

The purpose of cultivating students' Sports Core literacy is to promote students' better growth, so sports teaching activities need to have a certain effect. This needs to follow the principle of effectiveness, which requires regular evaluation of College Students' Sports Core literacy and understanding 
the problems in sports core literacy through evaluation [10]. In the evaluation, we should use a variety of evaluation indicators, including sports emotion and character, sports ability and habits, health knowledge and behavior, and so on. Through the effect evaluation, we found the problems in the cultivation of sports core literacy, and made targeted compensation and improvement to enhance the overall quality of students.

\subsection{Cultivation Strategy of Students' Sports Core Literacy}

\subsubsection{Improve Ideological Understanding}

In the process of cultivating students' Sports Core literacy, we need to change the concept of schools and students, and strengthen their attention to sports core literacy. In school, the evaluation mode should be changed. In the evaluation of students, we need not only evaluate through the students' academic performance, but also need to comprehensively investigate the students' Ideological and political level, sports core literacy, etc. Using these indicators can better understand the basic situation of students. At the same time, it is conducive to the students to invest more energy in the sports core literacy. The school can invite some experts and scholars to give lectures on Sports Core literacy from time to time. In the lecture activities, students and physical education teachers can communicate with experts and scholars, and inform experts and scholars of their understanding of sports core literacy, which is helpful for experts and scholars to make targeted explanation and enhance their understanding based on the existing mistakes in understanding. In terms of students, they should realize the importance of sports core literacy training for their own development. With the development of the times, the requirements for talents are constantly improved. The core quality of physical education is an important indicator to reflect the comprehensive ability of students. Students should consciously cultivate their own sports core literacy, actively participate in sports activities and cooperate with physical education teachers to ensure the smooth completion of teaching objectives.

\subsubsection{Optimize the Curriculum System}

Higher vocational colleges need to improve the physical education curriculum system, integrate the elements of sports core literacy into teaching objectives, and enrich the existing teaching content resources. First of all, we need to increase the number of physical education courses, from one physical education class a week to two classes a week, so as to expand the proportion of physical education. At the same time, we should optimize the teaching content. In the past, most of the teaching contents were single sports, which could not meet the needs of students. In order to improve the curriculum system, different types of physical education teaching can be established, such as confrontation module, competitive module and so on. Students can choose the appropriate module according to their own interests. Secondly, we need to take the cultivation of students' Sports Core literacy as the curriculum goal. The traditional physical education curriculum is to take the sports as the breakthrough, according to the students' physique and skills as the standard. In the new curriculum we puts forward new and higher requirements. The curriculum development should take the students' quality as the core, take the students' ability and morality as the goal, transform the physique and skills into tools, and pay attention to the students' endogenous learning. During the course, we should enhance students' interest in learning, enhance their emotional experience, pay attention to the cultivation of students' morality and ability, and help students establish the awareness of lifelong physical education. In the construction of physical education curriculum system, we also need to realize the connection between inside and outside the class and school, and establish a correct curriculum view. The traditional physical education curriculum is mainly concentrated in the school and classroom, and has not been extended to the society and community, so the classroom system is relatively incomplete. Colleges and universities can properly organize students to participate in some social competitions or social public welfare activities, which can not only enhance students' physical quality, but also contribute to social progress.

\subsubsection{Strengthen the Construction of Teaching Staff}

Physical education classroom is an important channel to cultivate students' Sports Core literacy. Physical education teachers are the organizers and promoters of classroom activities. The quality of physical education teachers is directly related to teaching interest and has a certain impact on students' learning interest [11]. Therefore, it is necessary to speed up the construction of teaching staff and provide support for the cultivation of students' Sports Core literacy. We can do in the following two ways. First, higher vocational colleges can carry on the training to the existing physical education teachers. Before the training of physical education teachers, we need to do a good job in the ability evaluation. Through the ability evaluation, we can understand the existing lack of physical education teachers' ability, and carry out targeted training in combination with the specific situation of teachers to improve the training effect. Second, physical education teachers should give full play to their autonomous learning ability, accumulate and supplement sports core literacy knowledge in their spare time, and enhance their overall quality. Physical education teachers also need to do a good job in the corresponding communication work, to optimize the existing teaching content and teaching arrangements. In communication, we should learn from each others' advantages and learn advanced teaching methods of other physical education teachers to make up for the deficiencies in their own ability. Physical education teachers should carefully study the policy and scientific research literature of sports core literacy, grasp the development trend and direction of physical education teaching, combine with the implementation of specific courses, find problems and solve problems in time, so as to clear the obstacles for implementing the cultivation means of College Students' Sports Core literacy in physical education curriculum. 


\subsubsection{Enrich Campus Sports Atmosphere}

Higher vocational colleges also need to create a good campus culture atmosphere to lay the foundation for the cultivation of sports core literacy. First of all, colleges and universities need to increase the investment in hardware resources, which can increase the opening of sports venues, provide the using of sports equipment, especially the construction of more basketball courts, volleyball courts, badminton courts, etc., and increase fitness equipment to ensure the material conditions for students to participate in sports, stimulate and guide students to participate in physical exercise, meet the needs of College Students' physical exercise, and encourage students to walk out of the dormitory and into the playground. Thirdly, normalize sports activities, such as the implementation of basketball games and football games in Colleges and departments, which can not only cultivate students' cooperation ability, enhance their comprehensive quality, but also enrich their spare time cultural life. In addition, colleges and universities can also set up corresponding associations to encourage students to participate in various sports associations, provide venues, funds and policy support for the development of sports society, expand the influence of sports associations, and create conditions for students to carry out sports activities in their spare time. Higher vocational colleges should establish corresponding campus culture, combine sports core literacy with campus culture, expand the content of campus culture, play the guiding role of campus culture, and cultivate students' sports concept of loving sports and sports [12].

\section{Conclusion}

The core quality of physical education is one of the focuses in the field of physical education in China. It is also an important quality that vocational students lack very much. It is an important factor that can not be ignored in the healthy growth and comprehensive development of higher vocational students [13]. However, there are some problems in the cultivation of students' Sports Core literacy in higher vocational colleges, including ignorance of the cultivation of sports core literacy, the imperfect curriculum system, the slow construction of teachers' team, and the need to further enrich the campus sports atmosphere, which requires higher vocational colleges to reform the physical education curriculum, construct the core objectives of physical education curriculum, cultivate the ability of physical education teachers, and verify the physical education to ensure that students master the basic knowledge and skills of physical education and health, enhance their physical fitness, enable them to learn the methods of physical education and exercise, develop their practice and innovation ability in sports and health activities, experience the fun and successful experience brought by sports to students, and develop good exercise habits for the students to lay the foundation for lifelong physical education, to ensure that every student can be healthy, harmonious and comprehensive development. To study the series of problems in the process of cultivation of students'
Sports Core literacy, we need to carry out the physical education teaching activities, select the appropriate theoretical basis and practical path, and further update the research methods and expand the research vision around the connotation definition, structural characteristics, system construction, practice exploration and other contents of sports core literacy [14].

\section{Acknowledgements}

Supported by "In 2020, Suining City Education and Sports Bureau ideological and political education special general topic."

\section{References}

[1] Notice of the State Council on printing and distributing the implementation plan of national vocational education reform (GF [2019] No. http://www.gov.cn/zhengce/content/2019-02/13/content 5365341.htm, 2019.2.13

[2] Central People's government, "decision of the Central Committee of the Communist Party of China on upholding and improving the socialist system with Chinese characteristics and promoting the modernization of national governance system and governance capacity", http://www.gov.cn/zhengce/2019-11/05/content 5449023.htm, 2019.11.5.

[3] Zhao Fuxue, Cheng Chuanyin. Guidance and cultivation of students' core competence in Physical Education [J]. Journal of Chengdu Institute of physical education, 2018, 4 (06): 104-109.

[4] Sun Hong, Zhou Jingguo, Lin Weixian. Research on the framework of core sports literacy of contemporary college students [J]. Journal of Hanshan Normal University, 2019, 40 (06): 98-104.

[5] Zhan Limin, Xu Jinzhi. Research on the core quality structure of College Physical Education [J]. Shandong sports science and technology, 2019, 41 (06): 66-69.

[6] Hou Jie. Research on the cultivation strategy of College Students' Sports Core literacy [D]. Tianjin Institute of physical education, March 1, 2020.

[7] Director of the research group on the core literacy of Chinese students' development answers the reporter's questions. China Education News Network, January 09, 2017.

[8] Guo Zhifeng. Research on the cultivation of College Students' Sports Core literacy in the new era [J]. Journal of culture, 2019 (12): 176-178.

[9] Tan Fenquan. Influencing factors and optimization path of College Students' Sports Core literacy cultivation [J]. Sports science and technology literature bulletin, 2019, 27 (12): 21-22+63.

[10] Meng Tao. Exploring the development strategy of College Physical Education and college students' core literacy [J]. Intelligence, 2019 (30): 186.

[11] Tian Chao. Current situation and Enlightenment of Cultivating College Students' Sports Core literacy [J]. Sports, 2018 (20): 44-45. 
[12] Zhang Weimin. Effective teaching strategies of junior middle school physical education from the perspective of core literacy $[J]$. Scientific consultation (education and scientific research), 2020 (8): 268.

[13] Wu Xiangning. Disccussion on the theoretical problems and realization path of students' Sports Core literacy in Higher Vocational Colleges [J]. Journal of Hubei Normal University (NATURAL SCIENCE EDITION), 2020 (01): 72-77.

\section{Biography}

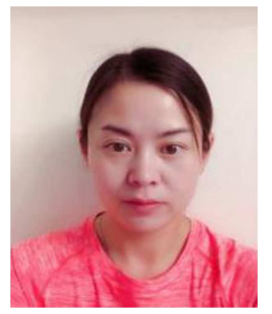

He Xiaoqin, 1985.01--, Lecturer, master of physical education; research direction: sports theory and sports training;Company: Sichuan Vocational and technical college, sports department, Suining, Sichuan, China.
[14] Zhao Fuxue, et al. Research on the core quality of physical education and its solutions [J]. Journal of physical education, 2019, 26 (6): 88-93.

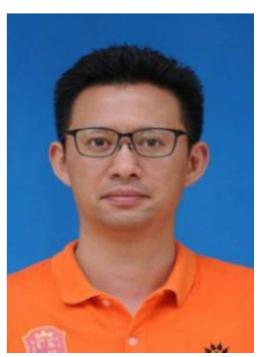

Zhu Chunquan, 1979.12--, associate professor, master of education; research direction: physical education teaching theory, sports policy and sports theory, sports training, etc; Company: Sichuan Vocational and technical college, sports department, Suining, Sichuan, China. 